\title{
Microfracture versus microfracture and platelet-rich plasma: arthroscopic treatment of knee chondral lesions. A two-year follow-up study
}

\author{
ANNALISA MANCÒ ${ }^{1}$, REMO GODERECCI ${ }^{1}$, ANNA RUGHETTI ${ }^{2}$, SILVANA DE GIORG ${ }^{3}$ \\ STEFANO NECOZIONE ${ }^{4}$, ALFREDO BERNARDI ${ }^{1}$, VITTORIO CALVISI ${ }^{1}$ \\ ${ }^{1}$ MESVA Department, Postgraduate School of Orthopaedics and Traumatology, University of L'Aquila, Italy \\ 2 Immunotrasfusional Unit, San Salvatore Hospital, ASL 1, L’Aquila, Italy \\ ${ }^{3}$ Department of Basic Medical Sciences, Neurosciences and Sensory Organs, University of Bari, Italy \\ ${ }^{4}$ MESVA Department, Clinical Epidemiology Unit, University of L'Aquila, Italy
}

\begin{abstract}
Purpose: the aim of this study was to describe and compare the clinical results obtained in patients affected by chondral lesions of the knee submitted to an arthroscopic treatment with the microfracture technique or microfracture + intraoperative autologous platelet-rich plasma (PRP) injection.

Methods: a prospective observational study was performed in patients affected by chondral lesions of the knee (classed as grade III-IV according to Outerbridge's classification) and early osteoarthritis (classed as grade 1-2 according to the Kellgren-Lawrence classification). Their mean age was 52.4 years. Thirteen patients were treated with the microfracture technique according to Steadman (Group A), while 14 were treated with microfracture + PRP injection (Group B). Both groups were assessed using series of measures (a visual analog scale for pain, the 36-Item Short Form Health Survey and the International Knee Documentation Committee Subjective Knee Form) to compare pre-operative and postoperative values at 3, 6, 12 and 24 months. Statistical analysis was conducted using a two-factor ANOVA for repeated measures.

Results: the VAS score decreased from a pre-operative value of $6.62 \pm 1.26$ to $3.54 \pm 2.26$ at 24 months in
\end{abstract}

\section{Corresponding Author:}

Annalisa Mancò, MD

MESVA Department, Postgraduate School of

Orthopaedics and Traumatology, University of L'Aquila

Via Monte Matese 7, 67100 L'Aquila, Italy

E-mail: annalisamanco@hotmail.com
Group A $(\mathrm{p}<0.001)$, and from $6.43 \pm 1.91$ to $3.36 \pm 2.84$ in Group B $(\mathrm{p}<0.001)$. The IKDC subjective score increased from a pre-operative value of $37.02 \pm 12.00$ to $62.13 \pm 19.00$ at two years in Group A $(\mathrm{p}<0.001)$ and from $34.63 \pm 15.00$ to $67.11 \pm 26.74$ in Group B $(\mathrm{p}<0.001)$; the SF-36 scores showed a similar trend. Although an improvement was recorded over time in both groups, in the short term the IKDC subjective score improvement seemed to be better in Group B; a similar trend was shown by the SF-36 and VAS scores. At two years, the IKDC Subjective Scale, VAS and SF36 scores seemed to be similar in the two groups. Over time, no significant differences were found between the two groups in any of the three outcomes.

Conclusions: the use of autologous PRP in association with the microfracture technique seems to give better clinical and functional results in short-term follow-up, above all as regards pain. At two-year followup, however, the clinical results of the two groups were similar.

Level of evidence: Level II, prospective cohort study.

Keywords: knee, cartilage, chondral lesions, microfracture, platelet-rich plasma (PRP).

\section{Introduction}

In orthopaedic practice, knee cartilage lesions are mainly diagnosed as a cause of pain and reduced joint function during daily activities and sports, usually in quite young subjects. Over the past two decades, many 
surgical techniques based on tissue engineering and biomaterials have been developed, and these have influenced the therapeutic algorithm for the treatment of cartilage disease. Today, surgical treatment options such as marrow stimulation techniques (e.g. the microfracture technique proposed by Steadman in 1997) are valid treatments for symptomatic focal articular cartilage defects $(1,2)$. Microfracture remains a first-line treatment option, whose advantages are minimal invasiveness, a single step, technical simplicity, low morbidity, and cost effectiveness. The technique involves perforating the subchondral bone plate, so as to allow cells from the bone marrow to repopulate defects, filling them with repair tissue (3). Histological and animal model studies have shown that the repair tissue obtained, called fibrocartilage, contains both type I and type II collagen (4). Its biomechanical and biological properties are inferior to those of normal articular cartilage, but it works as a filler of cartilage lesions that is able to resolve pain and restore joint function. These properties and the beneficial effects of this surgery have been variously debated in the literature over the years.

Platelet-rich plasma (PRP), used for the first time in maxillofacial surgery in 1998, has been, and continues to be, widely studied for its action on tissue regeneration and repair processes; it now occupies a very prominent place in medical practice and has different areas of application (5). It consists of platelets (PLTs) concentrated in a small volume of plasma that, once activated, degranulate, releasing bioactive factors (growth factors, GFs). These include platelet-derived growth factor (PDGF), transforming growth factor-b (TGF-b), platelet-derived epidermal growth factor (PEDGF), vascular endothelial growth factor (VEGF), insulin-like growth factor 1 (IGF-1), fibroblastic growth factor (FGF), and epidermal growth factor (EGF), each with a specific action at tissue level, playing a key role in chemotaxis, cell proliferation and inflammatory response modulation.

The definition and characterization of PRP is widely debated, with different methods of preparation resulting in differences in its PLT concentration and in the GFs it contains. For this reason, data on its efficacy are poorly comparable.

In vitro investigations show that PRP enhances mesenchymal stem cell proliferation and chondrogenic differentiation (6). In vivo investigations in an animal model (ovine) show that repeated injections of
PRP enhance the repair response after microfracture treatment, resulting in repair tissue with better macroscopic and histological features (7).

The aim of this study was to describe the clinical results of application of the microfracture technique in patients affected by chondral lesions of the knee and to evaluate whether combining intraoperative autologous PRP injection with microfracture improves the treatment outcomes. The hypothesis of the study was that PRP can improve the outcome of microfracture in the arthroscopic treatment of chondral lesions of the knee.

\section{Methods}

A prospective, observational study was carried out to evaluate the effectiveness of an arthroscopic treatment of chondral lesions of the knee (the microfracture marrow stimulation technique) compared with the same treatment administered in association with autologous PRP injection. Between 2011 and 2013, 27 patients affected by chondral lesions of the knee (all grade III-IV according to Outerbridge's classification) and early osteoarthritis (OA) (grade I-II according to the Kellgren-Lawrence classification) were enrolled. They had a mean age of 52.4 years and were followed up for a minimum of two years. Exclusion criteria were: major axial deviation (varus or valgus deformity $>5$ degrees), severe $\mathrm{OA}$, hematological diseases (coagulopathies), body mass index $>30$, lesion size $>4$ $\mathrm{cm}^{2}$, age $>60$ years, $\mathrm{Hb}<11.5 \mathrm{~g} / \mathrm{dL}$, PLT count $<100,000 / \mu \mathrm{L}$, tumors, infection, crystal arthropathies. The patients were divided into two groups: 13 patients (Group A) underwent arthroscopic microfracture and 14 patients (Group B) underwent arthroscopic microfracture and intrarticular injection of PRP (previously activated with $\mathrm{Ca}$ gluconate), the latter performed at the end of the arthroscopic procedure.

\section{PRP preparation}

Platelet-rich plasma (PRP), produced by the service of immunohemathology and transfusion medicine, was prepared using triple bags (Blood Bag, Terumo; Penpol Ltd, Trivandrum, India) and $150-200 \mathrm{ml}$ of autologous whole blood. The units of blood were fractionated to obtain: a) red blood cell concentrate (RBC); b) a hyperconentrate of PLTs suspended in 
10-15 ml of plasma (PRP); c) platelet-poor plasma (PPP). The fractionation process consisted of an initial centrifugation of the bag at $462 \mathrm{xg}$ for 10 minutes at $22^{\circ} \mathrm{C}$ in a centrifuge (Cryofuge 6000i; Hereaus Instruments AHSI Spa, Massa Martana, Italy) to separate out the red blood cells; this was followed by a second centrifugation at $3932 \times \mathrm{g}$ for 6 minutes at $22^{\circ} \mathrm{C}$ to obtain a PLT concentrate and PPP (plateletpoor plasma). The PLTs were finally collected as a "hyperconcentrate" in 10-15 ml of plasma at 0.3-1.5 $\mathrm{X} 10^{6}$ PLTs. The PRP thus obtained was divided into two aliquots of $6-8 \mathrm{ml}$ each and stored at $\leq 40^{\circ} \mathrm{C}$ for 12 months; one of these aliquots was for use, the other to be kept in reserve. To preserve its sterility, the PRP was activated with Ca gluconate (1:10-15) immediately before its intrarticular injection at the end of the arthroscopic procedure in the operating room.

\section{Surgical technique}

After arthroscopic diagnostic evaluation, a microfracture procedure according to Steadman's technique was performed by the same surgeon. All the unstable cartilage was removed and the cartilage lesion was prepared with debridement of the subchondral bone. After measuring the length and width of the lesion using a probe, its area was calculated in centimeters squared. Angled awls were used to make holes perpendicularly through the subchondral bone; these were $2-4 \mathrm{~mm}$ deep and were placed 3-4 $\mathrm{mm}$ apart. The arthroscopic inflow was closed and bleeding from the holes was verified (Fig. 1). In the group treated with PRP (Group

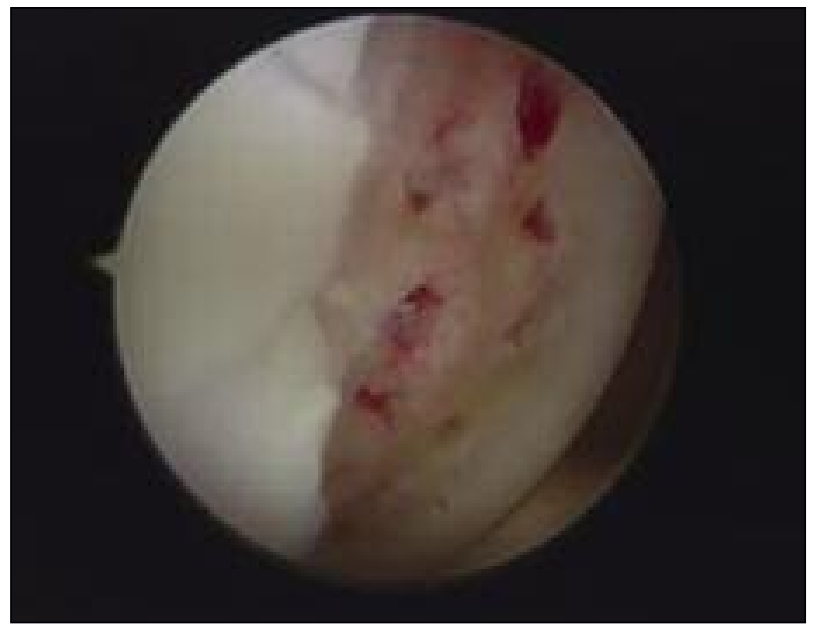

Fig 1. Steadman's microfracture (Group A).

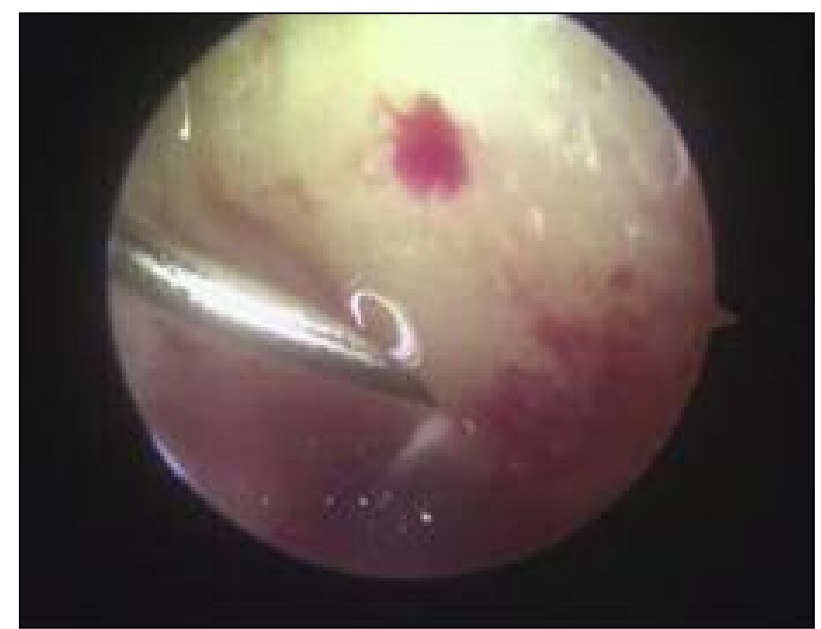

Fig 2. PRP injection at the end of arthroscopic treatment (Group B).

B), at the end of the microfracture procedure, in a dry conditions, 6-8 $\mathrm{ml}$ of Ca gluconate-activated PRP was injected into the joint, around the site of the lesion under arthroscopic view (Fig. 2). All the patients followed the same post-operative rehabilitation program, using crutches and avoiding weight bearing for 4 weeks, after which full weight bearing was gradually introduced. A physiotherapy program consisting of isometric exercises and active closed-chain exercises was started during the hospitalization and continued after discharge.

\section{Outcome measurements}

Clinical and functional outcome was assessed preoperatively and post-operatively at 3, 6, 12 and 24 months using the International Knee Documentation Committee (IKDC) Subjective Knee Form, and a patient-administered visual analog scale (VAS) to grade knee pain. The 36-Item Short Form Health Survey (SF-36) was used to assess health status over time.

\section{Data analysis}

All continuous data were expressed as means and standard deviations of the mean. The Shapiro-Wilks test was used to evaluate the normality of distributions of variables. A two-factor analysis of variance (ANOVA) for repeated measures, after logarithmic transformation of variables in the event of non-normality of data, was performed to assess differences between groups. Time effect, group effect and time by 
group interaction were investigated. P-values less than 0.05 were taken as statistically significant. A test of within-subjects effects was carried out with $p<0.05$ indicating the presence of a significant difference between repeated measurements. If "group by factor interaction" showed $\mathrm{p}<0.05$, the difference between

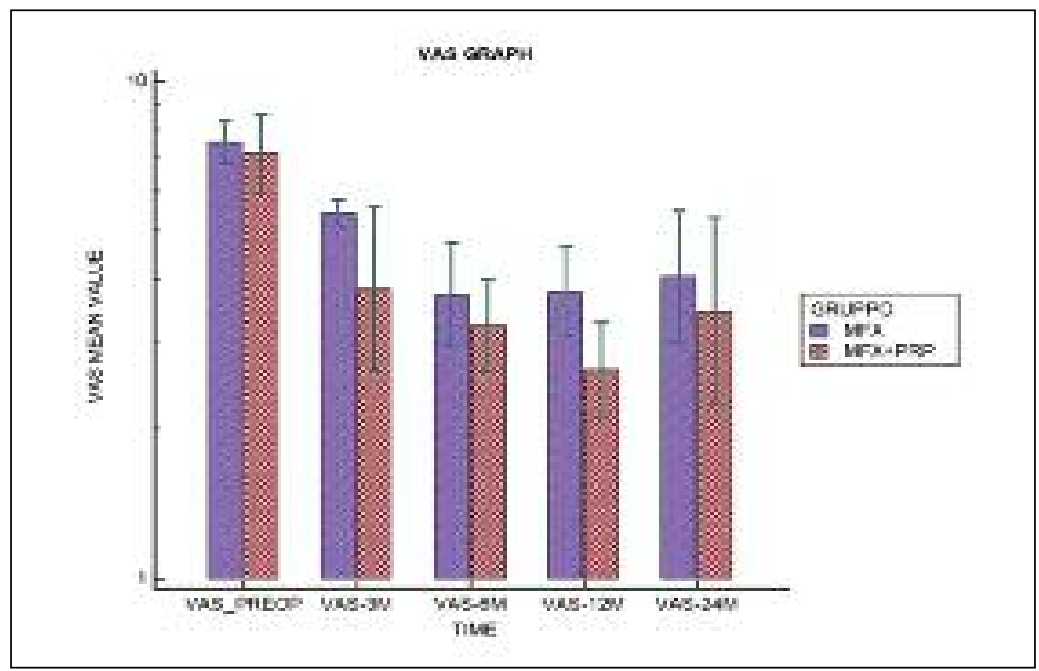

Fig 3. Distribution of VAS scores over time. measurements depended on group membership; pvalues greater than 0.05 indicated no significant difference in the comparison of the measurements over time between the two different treatment groups.

Statistical analysis was carried out using SAS System version 9.4 (SAS, Cary, NC, USA) and Med Calc statistical software version 13.3.1 (MedCalc Software bvba, Ostend, Belgium).

\section{Results}

No major adverse events were described after surgery. The clinical results of both groups at each follow-up are shown in Table 1.

In both groups, significant improvements were observed over time $(\mathrm{p}<0.001)$ in the VAS (Fig. 3), IKDC Subjective Scale (Fig. 4) and SF-36 (Fig. 5) scores, while no significant difference was observed on the comparison between the two groups in any of the three tests over time $(\mathrm{p}>0.05)$.

Table 1. Mean values with standard deviation of VAS, IKDC, SF-36 in both groups.

\begin{tabular}{|c|c|c|c|c|}
\hline VARIABLES & GROUP A & GROUP B & P-value for Time & $\begin{array}{l}\text { P-value for Group } x \\
\text { Time interaction }\end{array}$ \\
\hline \multicolumn{5}{|l|}{ VAS } \\
\hline PRE-OP & $6.62 \pm 1.26$ & $6.43 \pm 1.91$ & \multirow{5}{*}{$\mathrm{p}<0.001$} & \multirow{5}{*}{$p=0.469$} \\
\hline 3 MONTHS & $4.46 \pm 0.52$ & $3.50 \pm 2.35$ & & \\
\hline 6 MONTHS & $3.00 \pm 1.63$ & $2.43 \pm 1.22$ & & \\
\hline 12 MONTHS & $3.00 \pm 1.53$ & $1.79 \pm 0.89$ & & \\
\hline 24 MONTHS & $3.54 \pm 2.26$ & $3.36 \pm 2.84$ & & \\
\hline \multicolumn{5}{|l|}{ IKDC } \\
\hline PRE-OP & $37.02 \pm 12.00$ & $34.63 \pm 15.00$ & \multirow{5}{*}{$\mathrm{p}<0.001$} & \multirow{5}{*}{$\mathrm{p}=0.457$} \\
\hline 3 MONTHS & $37.40 \pm 10.83$ & $47.01 \pm 16.31$ & & \\
\hline 6 MONTHS & $58.05 \pm 11.90$ & $60.60 \pm 8.99$ & & \\
\hline 12 MONTHS & $62.58 \pm 9.60$ & $72.10 \pm 5.93$ & & \\
\hline 24 MONTHS & $62.13 \pm 19.00$ & $67.11 \pm 26.74$ & & \\
\hline \multicolumn{5}{|l|}{ SF-36 } \\
\hline PRE-OP & $48.07 \pm 15.00$ & $54.72 \pm 15.42$ & \multirow{5}{*}{$\mathrm{p}<0.001$} & \multirow{5}{*}{$\mathrm{p}=0.346$} \\
\hline 3 MONTHS & $48.35 \pm 17.77$ & $47.44 \pm 8.60$ & & \\
\hline 6 MONTHS & $68.71 \pm 15.92$ & $64.11 \pm 12.60$ & & \\
\hline 12 MONTHS & $72.84 \pm 15.00$ & $76.23 \pm 8.30$ & & \\
\hline 24 MONTHS & $66.13 \pm 22.00$ & $70.32 \pm 18.43$ & & \\
\hline
\end{tabular}




\section{Toints}

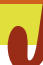

\section{Discussion}

Several studies published in the literature have shown a significant improvement of clinical results in a high percentage of patients treated with microfracture (70$90 \%$ ), especially in the performance of daily activities, but also in sports, with the best results obtained in the first two years after treatment $(8,9)$.

Other studies with longer follow-ups showed that after this time point there was a worsening of the results in

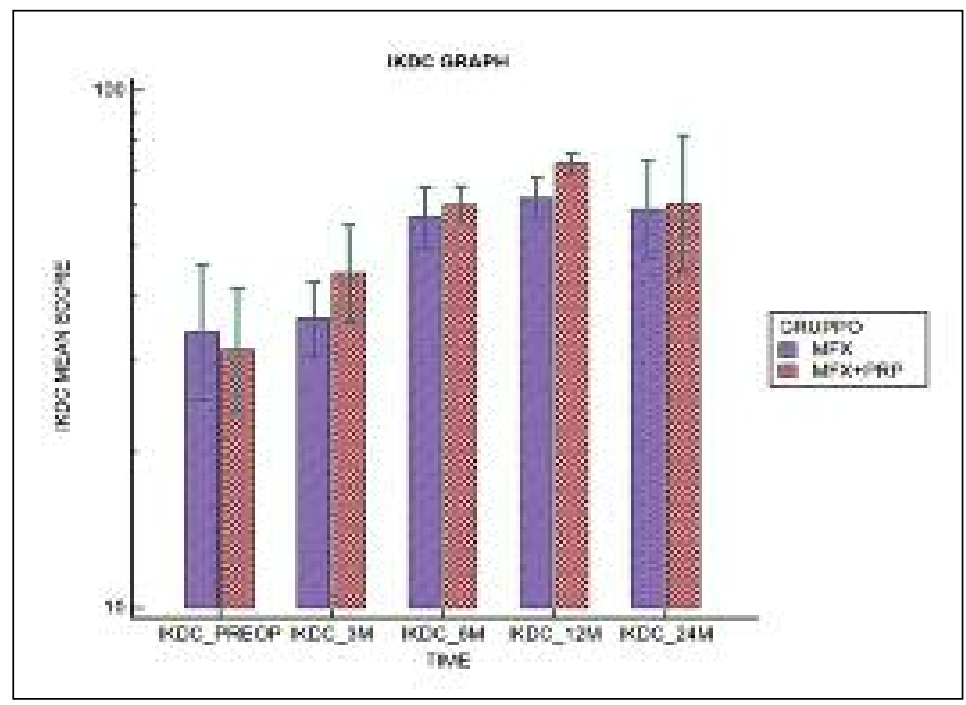

Fig 4. Distribution of IKDC scale scores over time.

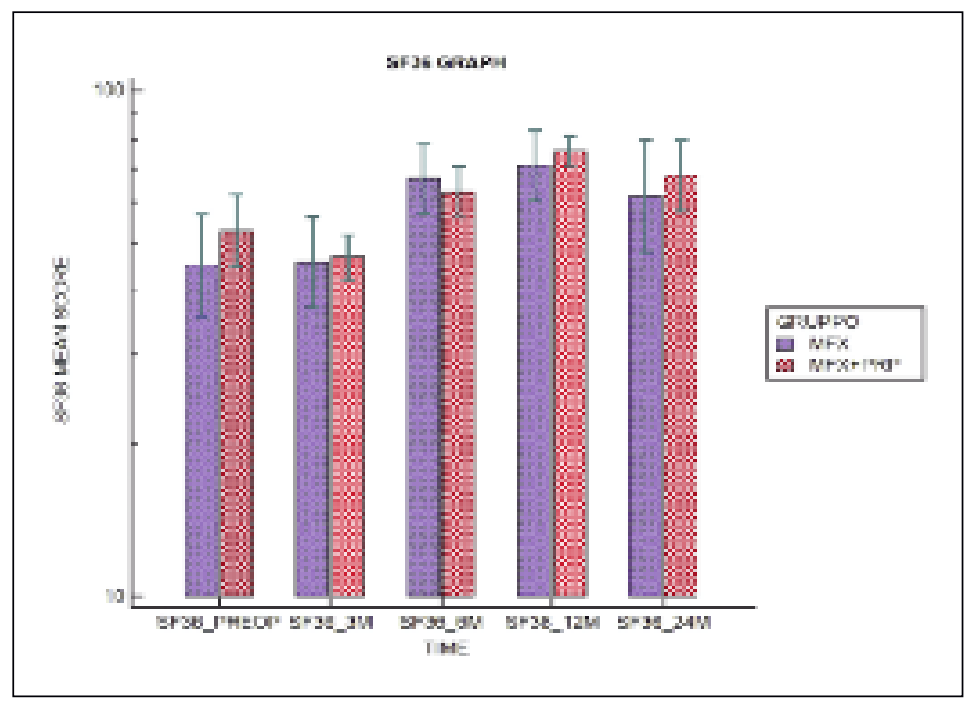

Fig 5. Distribution of SF-36 scores over time. terms of knee joint function, due to a lack of fibrocartilage tissue coverage of chondral lesions after treatment; they attributed the failure to poor execution of the technique $(10,11)$. More recently the comparison between bone marrow stimulation and other chondral regeneration techniques, such as autologous chondrocyte implantation (ACI), has shown comparable results between the two techniques after two years, but better clinical results in the group treated with second-generation ACI $(12,13)$. Solheim et al., in a long-term evaluation of the results of microfracture treatment (at 10-14 years), noted a worsening of the clinical outcome in $46 \%$ of patients (14). Bae et al. reported a decline in clinical improvement and a rate of "survival of microfractures" that declined from $89 \%$ at 5 years, to $68 \%$ at 10 years and $46 \%$ at 12 years (15). Even though the literature lacks objective criteria allowing standardization, and therefore better comparison, of the use of PRP in cartilage lesions, several studies seem to have shown the clinical efficacy of this approach in the short term, especially with regard to pain. In an in vivo animal model, Descalzi et al. postulated a potent antinociceptive activity of PRP related to endocannabinoid receptor activation in a mouse model of acute inflammatory pain (16). Pereira et al., in an in vitro study on primary human articular chondrocytes cultured under inflammatory conditions [comparable to those found in OA (+ IL-1 alpha)], initially showed a transient proinflammatory effect and then a drastic reduction of the expression of COX-2 and NB-kb activity, promoting resolution of the inflammatory process $(17,18)$.

In humans, the clinical efficacy of PRP in early OA has been variously studied. Kon et al. noted a short-term efficacy in reducing pain and improving both knee function and quality of life (19). A prospective, randomized, double-blind study evaluating the clinical efficacy of PRP in early OA compared to placebo (saline) noted, despite a general deterioration of the results after six months, better results, in terms of the effects on pain, stiffness and knee function, in patients treated with PRP (20).

A single study in the literature comparing microfracture alone with microfracture + 
PRP noted, contrary to our results, an improvement at two years in the group treated with (non-activated) PRP, and also an important effect on pain in the short term and macroscopic evidence of better quality cover tissue in arthroscopic second looks (21). These results, however, were not validated by histological investigation.

Analyzing our results, the subjects in Group B seemed to show a greater reduction of pain, resulting in improved joint function compared to Group A at the one-year follow-up. Over time, however, both groups showed a significant improvement of clinical outcomes compared to their preoperative conditions, and at two years their results were found to be similar. Thus, the efficacy of the microfracture technique as documented in our study appears to be totally in line with the current literature; indeed, microfracture, irrespective of whether it was associated with PRP use, produced a clinical improvement in terms of pain, knee function and general health, and this improvement was statistically significant over time $(p<0.001)$.

This study has several limitations, such as the small number of patients included, the short follow-up period, and the absence of second-look arthroscopies and histological analysis of the repair tissue. Despite these limitations, it confirmed that microfracture is a good option for relieving the symptoms of chondral lesions, and indicated that clinical outcomes are not affected by PRP injection. Further investigations including histological comparison and enrolling a greater number of patients could give us more definite answers regarding the possible effect of PRP injection on the well verified technique of microfracture.

\section{References}

1. Steadman JR, Rodkey WG, Singleton SB, et al. Microfracture technique for full-thickness chondral defects: technique and clinical results. Oper Tech Orthop. 1997;7:300-304.

2. Branca A, Calvisi V, et al. Metodiche conservative: tecniche artroscopiche (arthroscopic debridement, articular cartilage resurfacing). G.I.O.T. 2001;27:350-358.

3. Calvisi V, Pappalardo S, Niceforo A, et al. Evoluzione morfologica ed istologica del rigenerato fibrocartilagineo dopo condroabrasione. G.I.O.T. 1990;suppl vol XVI, n. 1:155/162.

4. Frisbie DD. Oxford JT, Southwood L, et al. Early events in cartilage repair after subchondral bone microfracture. Clin Orthop Relat Res. 2003;(407):215-227.

5. Marx RE, Carlson ER, Eichstaedt RM, et al. Platelet-rich plasma: growth factor enhancement for bone grafts. Oral Surg Oral Med Oral Pathol Oral Radiol Endod. 1998;85:638-646.

6. Mishra A, Tummala P, King A, et al. Buffered platelet rich plasma enhances mesenchymal stem cell proliferation and chondrogenic differentiation. Tissue Eng Part C Methods. 2009;15:431-435.

7. Milano G, Sanna Passino E, Deriu L, et al. The effect of platelet rich plasma combined with microfractures on the treatment of chondral defects: an experimental study in a sheep model. Osteoarthritis Cartilage. 2010;18:971-980.

8. Mithoefer K, Williams RJ 3rd, Warren RF, et al. The microfracture technique for the treatment of articular cartilage lesions in the knee. A prospective cohort study. J Bone Joint Surg Am. 2005; 87:1911-1920.

9. Knutsen G, Drogset JO, Engerbretsen L, et al. A randomized trial comparing autologous chondrocyte implantation with microfracture. Findings at five years. J Bone Joint Surg Am. 2007; 89:2105-2112.

10. Blevins FT, Steadman JR, Rodrigo JJ, et al. Treatment of articular cartilage defects in athletes: an analysis of functional outcome and lesion appearance. Orthopedics. 1998;21:761768.

11. Mithoefer K, McAdams T, Williams RJ, et al. Clinical efficacy of the microfracture technique for articular cartilage repair in the knee: an evidence-based systematic analysis. Am J Sports Med. 2009;37:2053-2063.

12. Kon E, Gobbi A, Filardo G, et al. Arthroscopic second-generation autologous chondrocyte implantation compared with microfracture for chondral lesions of the knee: prospective nonrandomized study at 5 years. Am J Sports Med. 2009;37: 33-41.

13. Kon E, Filardo G, Berruto M, et al. Articular cartilage treatment in high-level male soccer players: a prospective comparative study of arthroscopic second-generation autologous chondrocyte implantation versus microfracture. Am J Sports Med. 2011;39:2549-2557.

14. Solheim E, Hegna J, Inderhaug E, et al. Results at 10-14 years after microfracture treatment of articular cartilage defects in the knee. Knee Surg Sports Traumatol Arthrosc. 2016;24: 1587-1593.

15. Bae DK, Song SJ, Yoon KH, et al. Survival analysis of microfracture in the osteoarthritic knee: minimum 10-year followup. Arthroscopy 2013;29:244-250.

16. Descalzi F, Ulivi V, Cancedda R, et al. Platelet-rich plasma exerts antinociceptive activity by a peripheral endocannabinoid-related mechanism. Tissue Eng Part A. 2013;19:21202129.

17. Pereira RC, Scaranari M, Benelli R, et al. Dual effect of platelet lysate on human articular cartilage: a maintenance of chondrogenic potential and a transient proinflammatory activity followed by an inflammation resolution. Tissue Eng Part A. 2013;19:1476-1488

18. Pujol, JP, Chadjichristos C, Legendre F, et al. Interleukin-1 and transforming growth factor-beta 1 as crucial factors in osteoarthritic cartilage metabolism. Connect Tiss Res. 2008; 49:293-297.

19. Filardo G, Kon E, Buda R, et al. Platelet-rich plasma intraarticular knee injections for the treatment of degenerative cartilage lesions and osteoarthritis. Knee Surg Sports Traumatol Arthrosc. 2011;19:528-535.

20. Patel S, Dhillon MS, Aggarwal S, et al. Treatment with platelet-rich plasma is more effective than placebo for knee osteoarthritis: a prospective, double-blind, randomized trial. Am J Sports Med. 2013;41:356-364.

21. Lee GW, Son JH, Kim JD, et al. Is platelet-rich plasma able to enhance the results of arthroscopic microfracture in early osteoarthritis and cartilage lesion over 40 years of age? Eur J Orthop Surg Traumatol. 2013;23:581-587. 